\title{
In Situ PM-IRRAS Study of CO Adsorption on Au Surfaces: Solving the Puzzle
}

\author{
Yunxi Yao, b*, Long Chen ${ }^{\mathrm{b}}$, Xinchun $\mathrm{Mao}^{\mathrm{a}}$, Yifei Yang ${ }^{\mathrm{a}}$, Jun Chen ${ }^{\mathrm{a}}$, Linsen Zhou

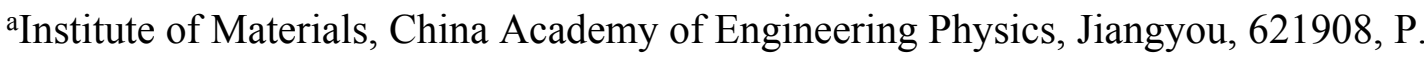 \\ R. China; \\ bDepartment of Chemistry, Texas A\&M University, College Station, Texas 77843, \\ USA \\ E-mail address: yaoyunxi@caep.cn (Y.Yao)
}

\section{Supporting Information}
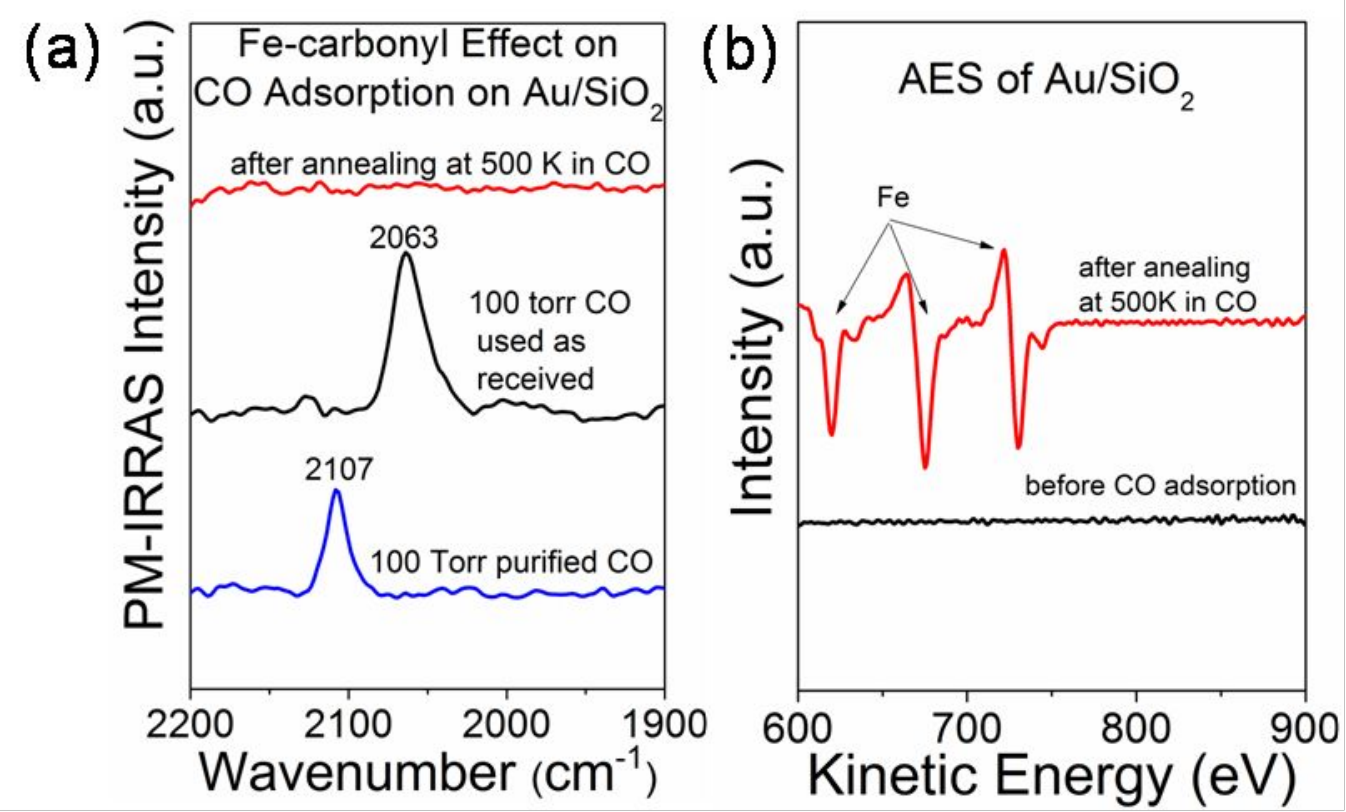

Figure S1. Iron carbonyl effect on $\mathrm{CO}$ adsorption on $\mathrm{Au} / \mathrm{SiO}_{2}$. (a) PM-IRRAS of CO adsorption on $\mathrm{Au} / \mathrm{SiO}_{2}$ prepared by depositing $5 \mathrm{MLE} \mathrm{Au}$ on $\mathrm{SiO}_{2} / \mathrm{Mo}(110)$. The bottom spectra are $\mathrm{CO}$ adsorption on $\mathrm{Au} / \mathrm{SiO}_{2}$ in 100 Torr $\mathrm{CO}$ with $\mathrm{CO}$ gas 
thoroughly purified, which shows a single $\mathrm{CO}$ adsorption peak at $2107 \mathrm{~cm}^{-1}$. The middle spectra show $\mathrm{CO}$ adsorption on $\mathrm{Au} / \mathrm{SiO}_{2}$ in 100 Torr $\mathrm{CO}$ with $\mathrm{CO}$ gas used as received without any purification. The $\mathrm{CO}$ adsorption peak at $2063 \mathrm{~cm}^{-1}$ is due to Fe-carbonyl adsorption on $\mathrm{Au} / \mathrm{SiO}_{2}$ or $\mathrm{CO}$ adsorption on the Fe deposits decomposed from Fe-carbonyl impurities in the $\mathrm{CO}$ gas. The top spectra recorded after annealing the $\mathrm{Au} / \mathrm{SiO}_{2}$ sample in the unpurified 100 Torr $\mathrm{CO}$ show no $\mathrm{CO}$ adsorption peaks. Annealing the $\mathrm{Au} / \mathrm{SiO}_{2}$ sample in high pressure $\mathrm{CO}$ environment results in $\mathrm{CO}$ dissociation on iron surface and iron oxide and carbide formation, which block CO adsorption on the surface.(b) AES of $\mathrm{Au} / \mathrm{SiO}_{2}$ before and after $\mathrm{CO}$ adsorption. The strong Fe AES peaks clearly indicate heavy Fe deposits on the surface decomposed from Fe-carbonyl impurities in the unpurified $\mathrm{CO}$ gas. We found $\mathrm{Fe}$-carbonyl from $\mathrm{CO}$ gas cylinder is easily completely removed by using a $\mathrm{LN}_{2}$ cooling trap, while $\mathrm{Ni}$-carbonyl in the $\mathrm{CO}$ gas is more difficult to be removed due to its higher vapor pressure. So if $\mathrm{CO}$ gas is purified by a $\mathrm{LN}_{2}$ cooling trap before $\mathrm{CO}$ adsorption experiments, the remaining metal-carbonyl in the $\mathrm{CO}$ gas, if any, is very likely Ni-carbonyl. 